\title{
Effect of inhaled corticosteroid particle size on asthma efficacy and safety outcomes: a systematic literature review and meta- analysis
}

Céline El Baou ${ }^{1,11^{*}}$, Rachael L. Di Santostefano ${ }^{2,9}$, Rafael Alfonso-Cristancho ${ }^{3,4}$, Elizabeth A Suarez ${ }^{5,10}$, David Stempel ${ }^{2}$, Mark L Everard ${ }^{6}$ and Neil Barnes ${ }^{7,8}$

\begin{abstract}
Background: Inhaled corticosteroids (ICS) are the primary treatment for persistent asthma. Currently available ICS have differing particle size due to both formulation and propellant, and it has been postulated that this may impact patient outcomes. This structured literature review and meta-analysis compared the effect of small and standard particle size ICS on lung function, symptoms, rescue use (when available) and safety in patients with asthma as assessed in head-to-head randomized controlled trials (RCTs).

Methods: A systematic literature search of MEDLINE was performed to identify RCTs (1998-2014) evaluating standard size (fluticasone propionate-containing medications) versus small particle size ICS medication in adults and children with asthma. Efficacy outcomes included forced expiratory volume in $1 \mathrm{~s}\left(\mathrm{FEV}_{1}\right)$, morning peak expiratory flow (PEF), symptom scores, \% predicted forced expiratory flow between 25 and $75 \%$ of forced vital capacity $\left(\mathrm{FEF}_{25-75 \%}\right)$, and rescue medication use. Safety outcomes were also evaluated when available.

Results: Twenty-three independent trials that met the eligibility criteria were identified. Benefit-risk plots did not demonstrate any clinically meaningful differences across the five efficacy endpoints considered and no appreciable differences were noted for most safety endpoints. Meta-analysis results, using a random-effects model, demonstrated no significant difference between standard and small size particle ICS medications in terms of effects on mean change from baseline $\mathrm{FEV}_{1}(\mathrm{~L})(-0.011,95 \%$ confidence interval $[\mathrm{Cl}]$ : $-0.037,0.014[\mathrm{~N}=3524])$, morning PEF $(\mathrm{L} / \mathrm{min})(\mathrm{medium} /$ low doses: $-3.874,95 \% \mathrm{Cl}:-10.915,3.166$ [N=1911]; high/high-medium doses: 5.551, 95\% Cl: $-1.948,13.049$ [N= 749])

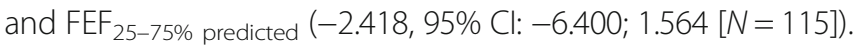

Conclusions: Based on the available literature, no clinically significant differences in efficacy or safety were observed comparing small and standard particle size ICS medications for the treatment of asthma.

Trial registration: GSK Clinical Study Register No: 202012.

Keywords: Inhaled corticosteroids, Particle size, Asthma, Systematic review, Meta-analysis

\footnotetext{
* Correspondence: celine.elbaou@phastar.com

${ }^{1}$ GSK, Middlesex, Stockley Park, Uxbridge, UK

${ }^{11}$ PHASTAR, Chiswick, London, UK

Full list of author information is available at the end of the article
} 


\section{Background}

Asthma is a common chronic lung condition characterized by inflammation of the airways, and defined by episodes of wheezing, chest tightness, shortness of breath, and coughing [1]. Treatment with regular daily inhaled corticosteroids (ICS) is highly effective at reducing symptoms and the risk of asthma exacerbation and is the primary therapy for control of chronic asthma in both adults and children [1]. The clinical effects of daily ICS are recognized in national and international guidelines as they eliminate or reduce chronic symptoms of asthma, prevent exacerbations, maximize lung function, reduce the need for rescue $\beta_{2}$-agonist treatment, and enable normal activity with few side effects at low and medium dose $[1,2]$.

Delivery of drug to the lungs is influenced by a number of factors including inspiratory flow and particle size. Current aerosol delivery systems generally deliver polydispersed aerosols with the majority of particles in the range $1-5 \mu \mathrm{m}$ in diameter [3]. Particles $<1 \mu \mathrm{m}$ are generally exhaled while most particles $>5 \mu \mathrm{m}$ are usually deposited in the upper airways. However, altering the characteristics of the aerosol even within this narrow window of 1-5 $\mu \mathrm{m}$ can alter the pattern of deposition within the lungs. As control of asthma by ICS requires delivery to both small and large airways, the differing particle size of ICS medications could potentially impact both efficacy and safety outcomes $[4,5]$. Traditional chlorofluorocarbon (CFC) pressurized metered dose inhalers (pMDIs) were all suspension-based formulations but following the CFC transition and the advent of hydrofluoroalkane (HFA) propellants, a variety of new suspension-based and solution-based formulations have been developed. Solution-based pMDIs differ from traditional suspension-based pMDIs in that the respirable particles are only generated after actuation as the propellant evaporates from the liquid plume [6,7]. The characteristics of the particles generated with solution-based pMDIs vary from formulation to formulation, with some generating extra-fine particles with mass median aerodynamic diameter (MMADs) of $<2 \mu \mathrm{m}$ while others generate particles with MMADs more comparable with traditional HFA-suspension pMDIs (MMADs of 2-5 $\mu \mathrm{m}$ ).

Two of the most widely prescribed ICS treatments are fluticasone propionate (FP) and beclometasone dipropionate (BDP), which are chemically and structurally similar but differ in their pharmacodynamic properties [5]. For patients not controlled on ICS alone, both the United States and European guidelines recommend the additional use of a long-acting $\beta_{2}$-agonist (e.g. salmeterol, formoterol, etc.) in a fixed-dose combination device. FP and FP/salmeterol (FP/SAL) are formulated as HFA-suspensions, while BDP, BDP-formoterol (BDP-F), and a more recent ICS, ciclesonide (CIC) are formulated as HFA-solutions which generate extra-fine aerosols [5]. Thus, FP and FP/SAL are considered standard particle size ICS $(2-5 \mu \mathrm{m})$, while BDP, BDP-F and CIC are considered small particle ICS $(<2 \mu \mathrm{m})$.

It has been postulated that the use of ICS medications with a smaller particle size may confer additional clinical benefits to patients with asthma compared with medications with particles of a standard size as they are able to access the smaller airways resulting in increased efficacy [8].

The objective of this systematic literature review and meta-analysis was to evaluate the impact of particle size on clinical outcomes of patients with asthma by comparing the effect of small and standard size particle ICS on lung function, symptoms, rescue use (when available) and safety as assessed in head-to-head randomized controlled trials (RCTs).

\section{Methods}

Details on the methods of the analysis and inclusion criteria were specified in advance and documented in a protocol (GSK Clinical Study Register ID: 202012, data on file), and are summarized below.

\section{Inclusion criteria, information source, search and study selection}

Studies eligible for inclusion in the systematic review were published RCTs comparing FP-containing therapy (standard particle size) with ICS preparations of small particle size in adults and children with asthma. Specifically, treatments evaluated included FP and FP/SAL versus ICS small particle size comparators (BDP, BDP-F or CIC). Abstracts for potential inclusion in the systematic review were identified from the MEDLINE database using the following search terms in PubMed: disease: asthma; exposure: fluticasone, Flovent ${ }^{\circ}$, Flixotide ${ }^{\ominus}$, Advair ${ }^{\circ}$, Seretide ${ }^{\circ}$. Abstracts in English published between January 1, 1998 and January 13, 2014 were considered.

All identified citations were downloaded and duplicate citations were removed to yield a number of unique hits. Citations were assessed in a multi-stage screening process as outlined in Fig. 1. During Screening Stage One, studies/abstracts were excluded if they included only patients with allergic rhinitis, compared ICS medications other than FP or FP/SAL versus BDP, BDP-F, or $\mathrm{CIC}$, were placebo-controlled, were not a primary epidemiologic/clinical study or were considered 'gray literature' (meeting abstracts, letters, websites). Other exclusion criteria included: restricted population (e.g. pregnant women); comparisons of the same ICS at different dosages; no efficacy or safety data. Citations were designated as 'Exclude,' 'Include' or 'Doubt' and a record of these decisions was maintained. Abstracts marked as 'Doubt' were cross-reviewed by a second epidemiologist. 


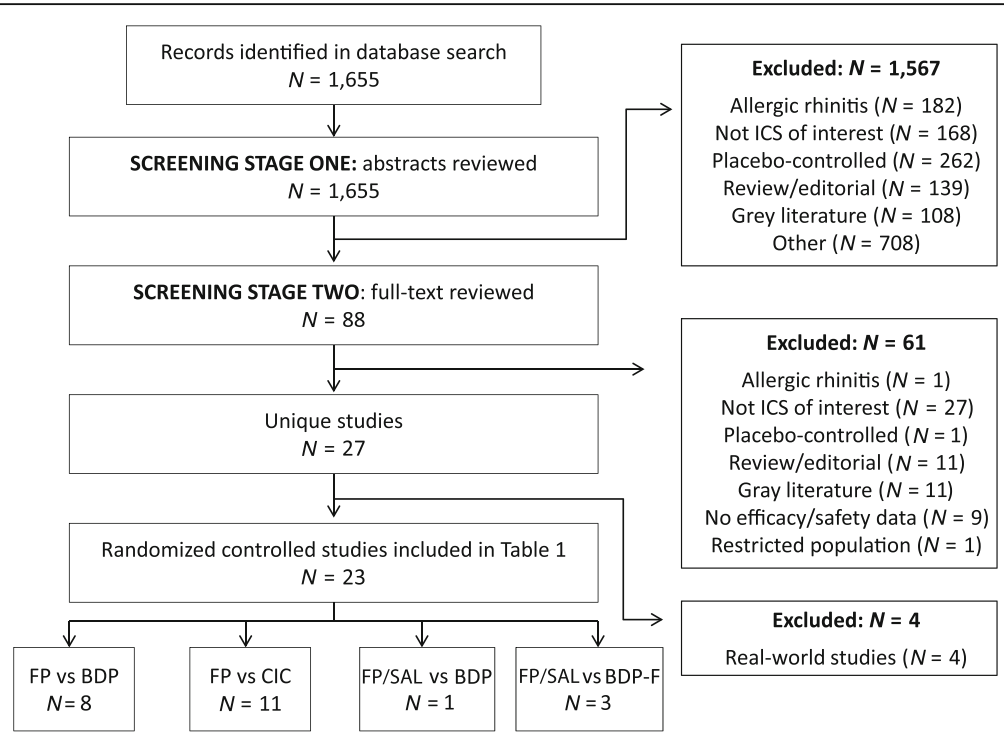

Fig. 1 Flow diagram showing implementation of search and screening strategies. BDP, beclometasone dipropionate, BDP-F, beclometasone dipropionate/formoterol fumarate; CIC, ciclesonide; FP, fluticasone propionate; FP/SAL, fluticasone propionate/salmeterol; ICS, inhaled corticosteroid

In Screening Stage Two, full text articles of the titles identified as 'Include' in Screening Stage One were reviewed and screened against the exclusion criteria listed above. The remaining studies were utilized for extraction.

\section{Data extraction and data items}

Study/patient characteristics and interventions were abstracted from the selected studies. Information on the following efficacy outcome measures were also extracted: forced expiratory volume in $1 \mathrm{~s}\left(\mathrm{FEV}_{1}\right)$, morning peak expiratory flow (PEF), asthma symptom scores (on 4-9-point scale where a lower score corresponded to fewer symptoms), \% predicted forced expiratory flow between 25 and $75 \%$ of forced vital capacity (FVC; $\mathrm{FEF}_{25-75 \%}$ ), and rescue medication use per day. In addition to the common lung function measure in asthma studies ( $\mathrm{FEV}_{1}$ and $\mathrm{PEF}$ ), $\mathrm{FEF}_{25-75 \%}$ was chosen as an efficacy measure as it is a more sensitive indicator for small airway obstruction than $\mathrm{FEV}_{1}$ [9], and thus more likely to demonstrate variations in efficacy if the smaller particles were meeting the small airways. To characterize available safety data, the following endpoints were considered: any adverse events (AEs; at least one), local steroid effects (oral candidiasis, hoarseness), upper respiratory tract infections, growth and bone metabolism, and serum cortisol levels to assess adrenal suppression.

\section{Assessment of risk of bias}

Funnel plots were used to detect biases in the identification and selection of studies. The funnel plot is a technique used to investigate the possibility of biases in the identification and selection phases. In a funnel plot, the estimated effect size of the intervention from individual studies (mean difference) is plotted on the horizontal axis against the standard error of the intervention effect estimate or sample size on the vertical axis. If there are no biases, the graph will tend to have a symmetrical funnel shape centered on the average effect of the studies. All studies were included and additional sources of bias were not formally assessed.

\section{Planned analysis and statistical methods}

Aggregated clinical data from the completed systematic review were summarized in standardized electronic extraction forms, with comparative data also entered into spreadsheets. Clinical statisticians transferred relevant extracted data into SAS (Statistical Analysis Software, Cary, NC) or R (R Foundation for Statistical Computing, Vienna, Austria) for calculation of appropriate statistics and data displays.

The objective of this analysis was to determine if there were any clinically significant differences in the comparative efficacy or safety of FP-containing medications with smaller particle ICS-containing comparators; this was evaluated in the form of a benefit-risk interval plot and/or meta-analysis, when appropriate. The data were extracted on the intent-to-treat (ITT) population as defined in each individual trial. The original publications gave treatment doses as either emitted or delivered; these same doses were reported within this manuscript for consistency. 
Treatment comparisons were made using absolute treatment differences between FP-containing formulations and small particle ICS, including 95\% confidence intervals (CI). For continuous measures, adjusted mean differences were used, when available. When standard errors (SE) and/or CIs were not directly available, they were estimated using available data [10]. For binary measures, the absolute risk difference and its $95 \% \mathrm{CI}$ were calculated using the normal approximation to the binomial distribution.

Formal meta-analysis was conducted for efficacy endpoints when there was sufficient sample size and homogeneity across trials. Due to this approach, there was no adjustment for multiple testing. If there was no significant evidence of heterogeneity across the studies, both fixed and random effects models were performed. The statistical heterogeneity of the meta-analysis was assessed by means of the Cochran Q, chi-square test and the I2 statistic with 95\% CI. When the assessments such as Cochran Q or chi-square test showed that heterogeneity existed, the results of the random effects model were selected. Results in children (12 years and younger) and adolescents/adults were analyzed separately. Meta regression was used to adjust for differences across studies as appropriate. Additional sensitivity analyses were performed when appropriate.

Where meta-analysis was not feasible, benefit-risk interval plots were produced to visually display the estimated differences between treatments and their 95\% CIs for different endpoints on the same graph across studies; irrespective of differences in study designs, endpoints and units.

\section{Results}

The search of the PubMed database identified 1655 potentially relevant articles: 1567 were excluded, mainly because they were placebo-controlled, evaluated allergic rhinitis or did not evaluate an ICS of interest; 88 fulltext articles were reviewed and 23 RCTs were included in the final analysis (Fig. 1) [4, 11-32].

Eight studies evaluated FP versus BDP, 11 evaluated $\mathrm{FP}$ versus $\mathrm{CIC}$, one evaluated $\mathrm{FP} / \mathrm{SAL}$ versus $\mathrm{BDP}$ and three evaluated FP/SAL versus BDP-F (Table 1). No studies evaluating FP versus BDP-F and FP/SAL versus $\mathrm{BDP}$ or CIC were identified. Information for children (6 to 15 years in age) was only available in four studies $[15,18,26,27]$; one of which utilized a spacer in each arm [18]. No other studies (adults or children) were found to use a spacer.

The main efficacy endpoints evaluated in the studies were $\mathrm{FEV}_{1}$ and PEF (Table 1). The predominant safety endpoints were overall incidence of AEs and urinary cortisol levels.

\section{Fluticasone propionate versus beclometasone dipropionate}

In the eight identified trials comparing conventional suspension-based FP pMDIs with the 'ultra fine' solutionbased BDP formulations similar doses have been used in each arm (Table 1). This is in accordance with GINA guidelines reporting the clinically comparable doses of HFA-FP and HFA-BDP [1]. Hence these comparisons will address the issue of whether differences in particle size results in a change in the efficacy or safety profile.

The majority of the RCTs reported no significant difference in efficacy outcome measures between FP and BDP. Two of the eight RCTs reported significant differences in $\mathrm{FEF}_{25-75 \%}$ between $\mathrm{FP}$ and BDP; one demonstrating improvement with FP [18] and the other with BDP [16] (Table 1).

The majority of RCTs reported no significant difference in AEs or other safety markers between the two treatments. Overnight urinary cortisol/creatinine production was suppressed more in patients using BDP compared with patients on FP $(1000 \mu \mathrm{g} /$ day FP versus BDP: geometric mean fold difference 1.97 [95\% CI: $1.28,3.02$ ]; $p<0.05$ ) [12]. No significant difference was found in cortisol levels in three studies, either in levels at the end of the treatment period or in change from baseline $[11,13,14]$.

\section{Fluticasone propionate versus ciclesonide}

With few exceptions in 10 identified RCTs, CIC was found to be non-inferior or not statistically different from FP for numerous efficacy endpoints (Table 1). Notably, in a trial by Pedersen et al. in children aged 6-11 years, non-inferiority of CIC to FP ( $88 \mu \mathrm{g}$ twice daily) was observed with regard to change in $\mathrm{FEV}_{1}$ from baseline over 12 weeks of treatment with $160 \mu \mathrm{g}$ but not $80 \mu \mathrm{g}$ once daily [27]. A similar trial in children aged $6-15$ years comparing the same doses found that CIC did not show non-inferiority in the change from baseline in morning or afternoon PEF following 12 weeks of treatment [26]. One trial of adult patients, by Cohen et al., found greater improvement in lung function among patients receiving FP compared with patients receiving CIC, specifically in mean \pm SD \% predicted $\mathrm{FEV}_{1}(0.5 \pm 4.3$ versus $-3.0 \pm 4.6$, respectively; $p=0.021)$ and $\mathrm{FEF}_{25-75 \%}(0.6 \pm 5.6$ versus $-3.6 \pm 6.0$, respectively; $p=0.034$ ) [4].

Results for differences in urinary cortisol levels (adjusted for creatinine) were variable, with greater adrenal suppression among patients receiving FP (compared with CIC) reported in an ITT analysis restricted to patients with normal creatinine levels $(p=0.006)$ [26]. In a small crossover study, patients on FP $2000 \mu \mathrm{g} /$ day had significantly lower mean overnight 10 -h urinary cortisol than patients on CIC (CIC versus FP: geometric mean fold difference 1.5 [95\% CI: 1.1, 2.0]; $p<0.05$ ) [24]. One 


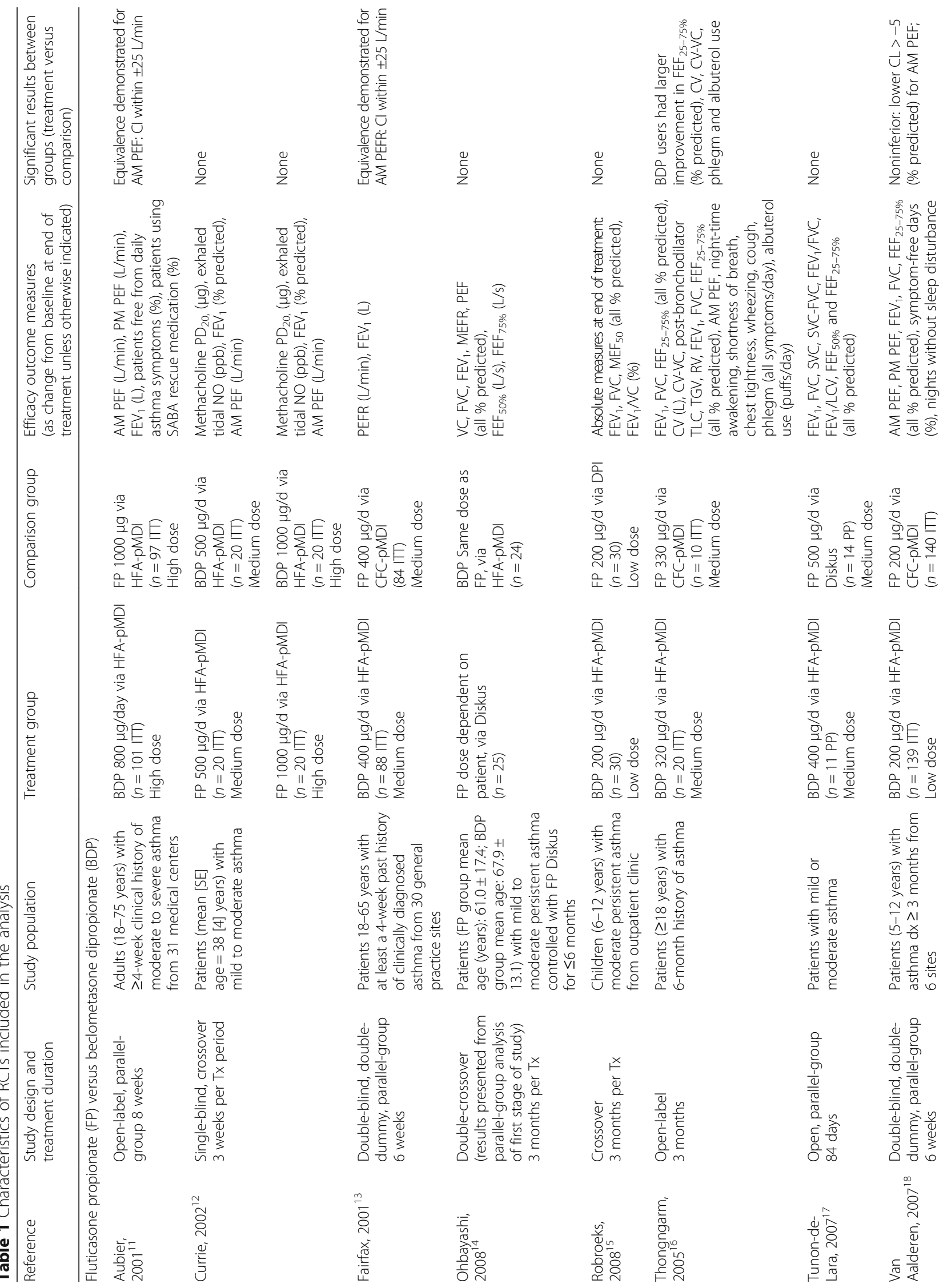




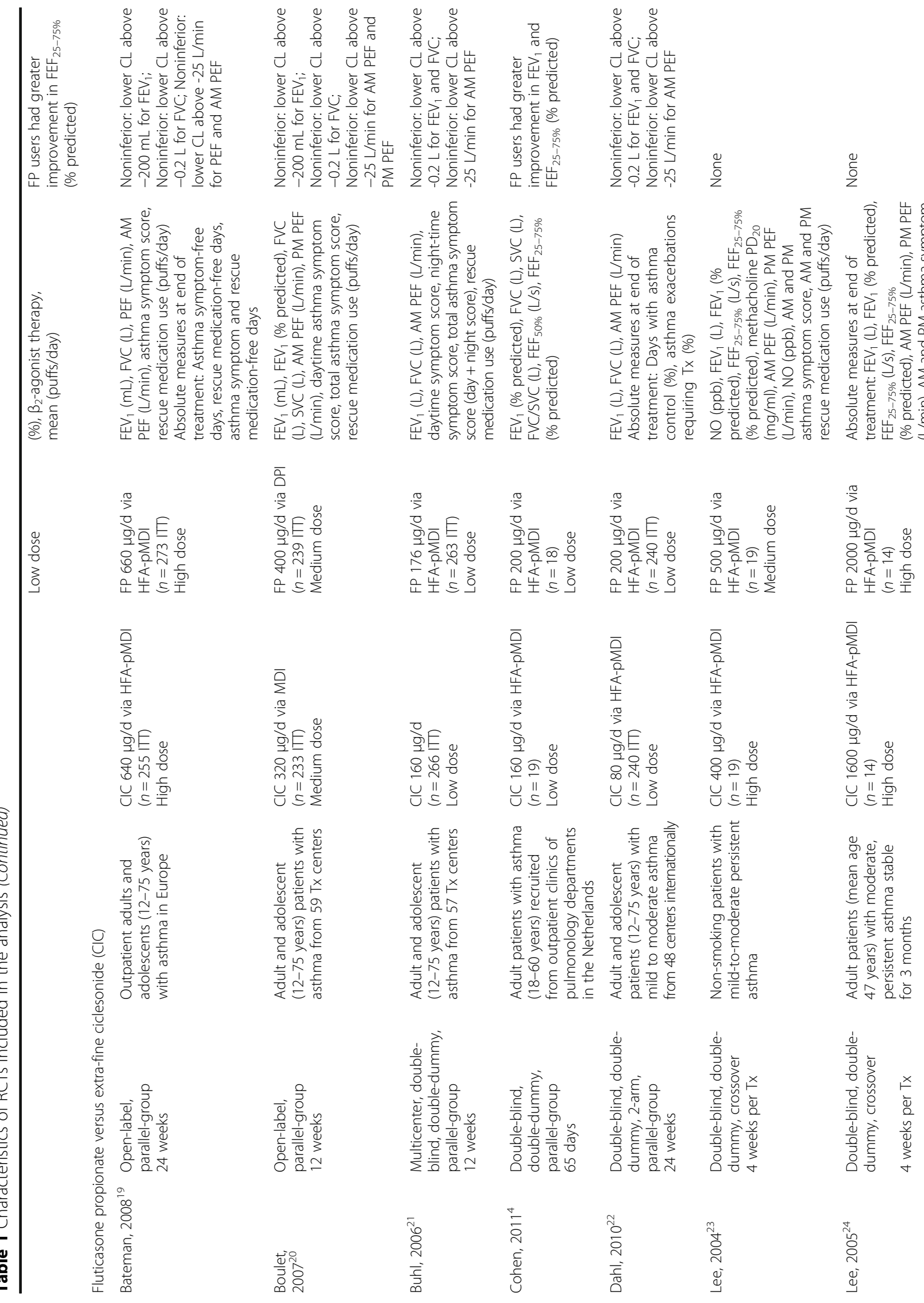




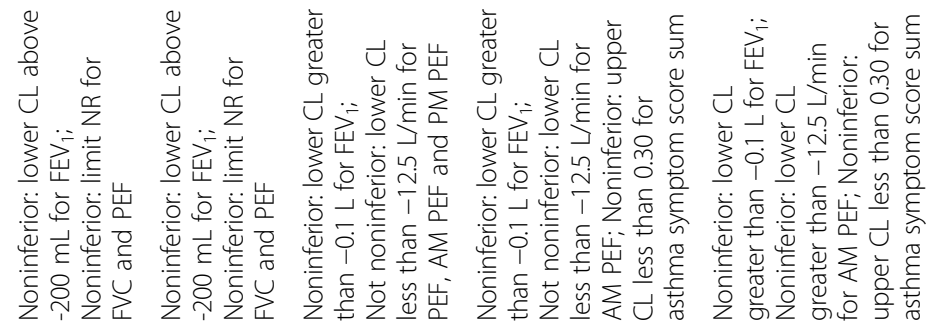

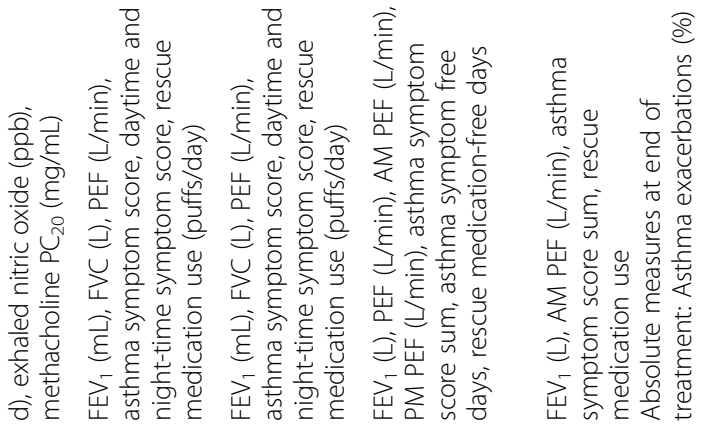

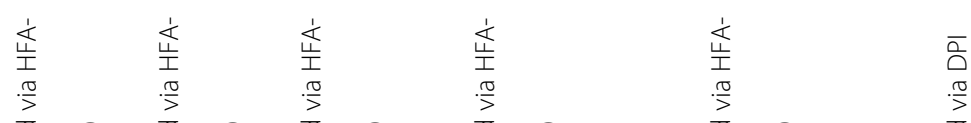

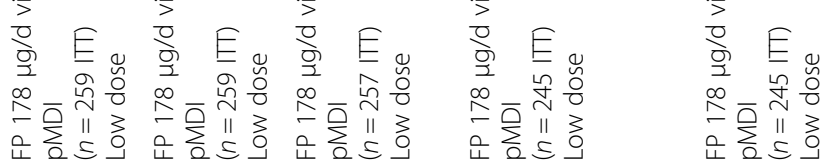

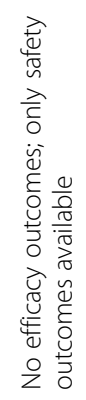

$\overline{2}$
$\overline{0}$
$\overline{0}$
$\frac{0}{2}$

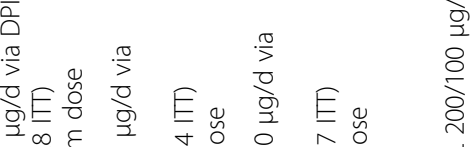

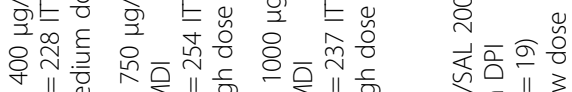

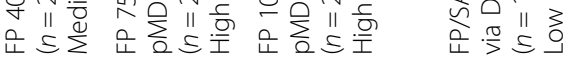

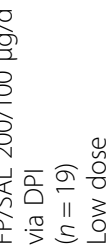

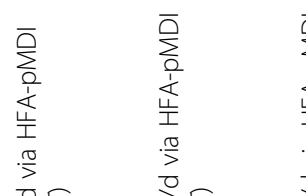

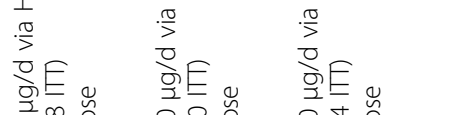

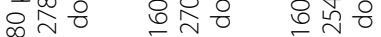

$\underline{\cup} \stackrel{\Perp}{\leq} 3$

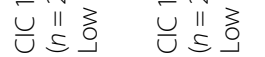

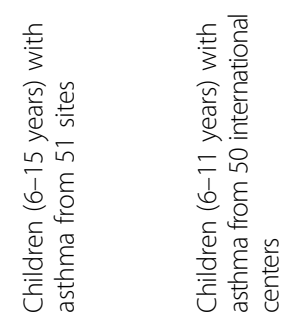

$\frac{\frac{1}{0}}{\frac{0}{0}}$

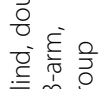

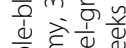

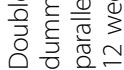

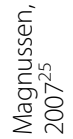

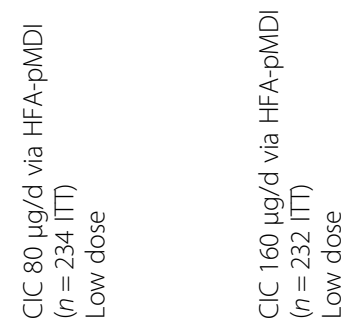

$\sum_{i}^{\bar{Q}} \quad \sum_{0}^{\overline{0}}$

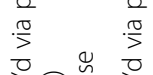

के트융 के

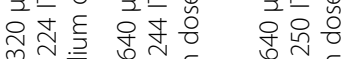

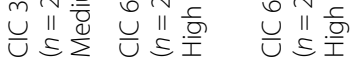

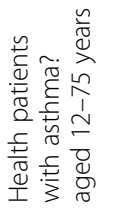

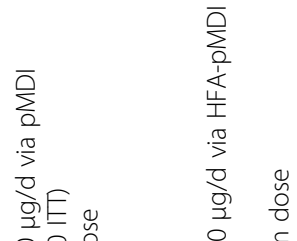

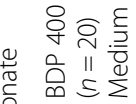

잉

․ㅡㄹ

芒

은 辰

पू山

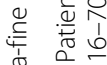

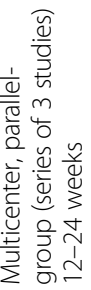

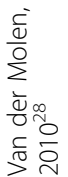

뭉
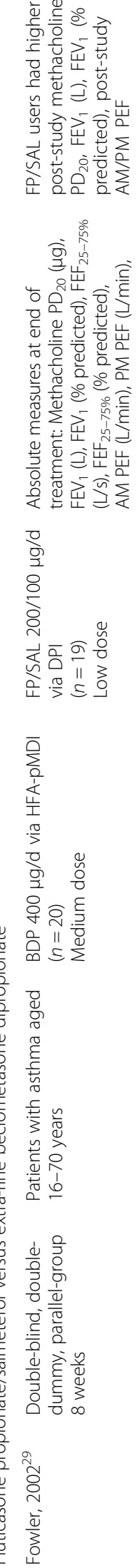
El Baou et al. BMC Pulmonary Medicine (2017) 17:31

Page 8 of 16

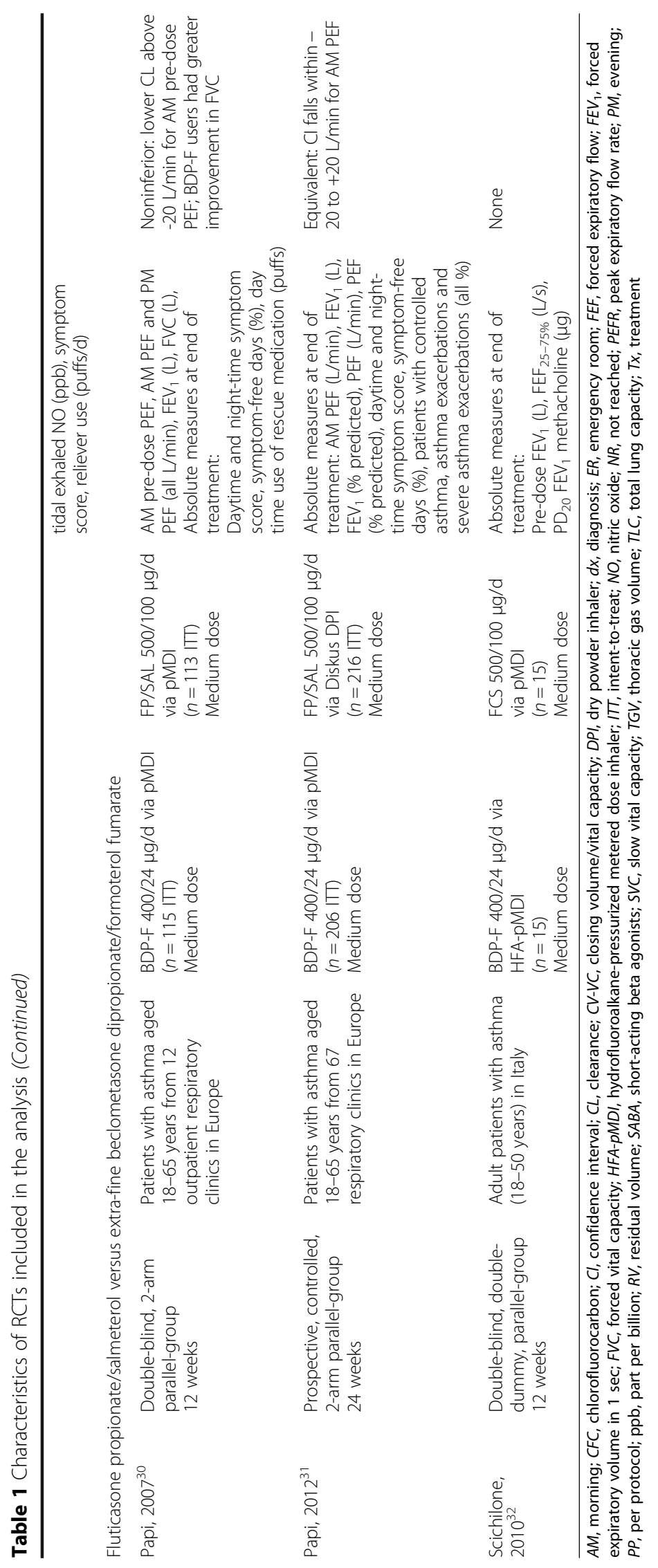


trial assessed side effect perception using a 100-point scale, and observed that patients on CIC had either a smaller increase in perceived side effects or a decrease over the treatment period from baseline compared with patients receiving FP (between-treatment least squares mean $( \pm \mathrm{SE})$ in total Inhaled Corticosteroid Questionnaire Scores: CIC $320 \mu \mathrm{g}$ once-daily versus FP $200 \mu \mathrm{g}$ twice-daily (12 weeks): $-2.52 \pm 0.82, p=0.0011$; CIC $320 \mu \mathrm{g}$ twice-daily versus FP $500 \mu \mathrm{g}$ twice-daily (24 weeks): $-2.05 \pm 0.79, p=0.0047$ [28].

\section{Fluticasone propionate/salmeterol versus beclometasone dipropionate}

One trial was identified that compared the efficacy of $\operatorname{BDP}(400 \mu \mathrm{g} /$ day $)$ and FP/SAL $(200 \mu \mathrm{g} / 100 \mu \mathrm{g} /$ day $)$ as a step-down therapy after high-dose ICS (dry powder inhaler [DPI]-BDP $2000 \mu \mathrm{g} /$ day) (Table 1) [29]. Lung function measures were compared between treatment groups at the end of the 8-week treatment period instead of comparing the change from baseline in each group. Methacholine $\mathrm{PD}_{20}$, post-study $\mathrm{FEV}_{1}$ (measured and \% of predicted), morning and afternoon PEF were all significantly greater in patients on FP/SAL than in patients on BDP (methacholine $\mathrm{PD}_{20}(\mu \mathrm{g})$ : 149.9 [95\% CI: 114.3, 196.5] versus 71.2 (95\% CI: 54.7, 92.8]; $\mathrm{FEV}_{1}$ (L): 2.46 [95\% CI: 2.39, 2.53] versus 2.26 [95\% CI: $2.20,2.33]$, $p<0.05 ; \mathrm{FEV}_{1}$ (\% predicted): 77 [95\% CI: 75, 79] versus 70 (95\% CI: 68, 72], $p<0.05$; morning PEF (L/min): 434 [95\% CI: 424, 445] versus 402 [95\% CI: 391, 411], $p<0.05$; evening PEF (L/min): 436 [95\% CI: 425, 446] versus 408 [95\% CI: 398, 418]; $p<0.05$ ). No differences were found in $\mathrm{FEF}_{25-75 \%}$, symptom scores, or reliever medication use [29]. No significant differences were found between treatment groups for serum cortisol levels, urinary cortisol/creatinine ratio, or serum osteocalcin [29].

\section{Fluticasone propionate/salmeterol versus beclometasone dipropionate-formoterol}

Three RCTs compared the efficacy of FP/SAL with BDP-F (Table 1) [30-32]. One trial in adults with asthma found significantly greater improvement in FVC in patients receiving $\mathrm{BDP}-\mathrm{F}$ than in those receiving $\mathrm{FP} / \mathrm{SAL}(0.46$ $\pm 0.51 \mathrm{~L}$ versus $0.34 \pm 0.44 \mathrm{~L}$, respectively; $p=0.040$ ); however, no differences were found for any other efficacy parameters [30].

Two RCTs were identified that compared the safety of BDP-F with FP/SAL; no differences in AEs or urinary cortisol/creatinine ratio were observed between the two treatments [30, 31].

\section{Meta-analysis}

Meta-analysis methods could only be applied for the efficacy endpoints of $\mathrm{FEV}_{1}, \mathrm{PEF}$, and $\mathrm{FEF}_{25-75 \%}$ (Fig. 2). Other efficacy and safety endpoints were not considered for the meta-analysis due to heterogeneity, potential publication bias, and disparity of endpoint definitions and/or timing of collection. In adults, the random effects models showed no significant differences between small and standard size particle ICS for change in $\mathrm{FEV}_{1}$ $(-0.011$ L, 95\% CI: $-0.037,0.014 ; p=0.394)$, or $\mathrm{FEF}_{25-75 \%}$ $(-2.418,95 \%$ CI: $-6.400,1.564 ; p=0.234)$ (Figs. 2a and c).

Meta-regression analysis showed that the only treatment effect modifier present for morning PEF was dose level (high versus low); however, high dose versus medium dose or high hose versus high-medium dose did not show a statistically significant difference. This suggested that it may not be appropriate to use either metaregression or meta-analysis with all of the data in the final model. Instead, the morning PEF endpoint was analyzed as two separate subgroups (high/high-medium doses and medium/low doses). The random effects models showed no significant differences between small and standard size particle ICS for change in morning PEF (medium/low doses: $-3.874 \mathrm{~L} / \mathrm{min}, \quad 95 \% \mathrm{CI}$ : -10.915, 3.166; high/high-medium doses: $5.551 \mathrm{~L} / \mathrm{min}$, 95\% CI: -1.948 , 13.049) (Figs. 2bi and ii).

For each endpoint, the heterogeneity test showed that there was no between-study variation, suggesting that fixed effects models were also appropriate. The random effects models, which provide a more conservative approach, were retained as primary models. Similar to the random effects model, no significant differences were observed for change in $\mathrm{FEV}_{1}$ and morning PEF using a fixed effects model ( $p=0.394$ and $p=0.097$, respectively). Even though the analysis of both data subgroups for the morning PEF data led to the same conclusion, the results for the mean differences between medium/low $(-4.223 \mathrm{~L} / \mathrm{min})$ and high/high-medium $(5.551 \mathrm{~L} / \mathrm{min})$ dose levels appeared to be in opposite directions (Figs. 2bi and ii). Treatment differences in $\mathrm{FEF}_{25-75 \%}$ were found to be significantly in favor of FP using a fixed effects model $(-2.853 \mathrm{~L} / \mathrm{min}$; 95\% CI -5.579 , $-0.127 ; p=0.040)$, though not in the random effects model $(-2.418,95 \%$ CI: $-6.400,1.564 ; p=0.234)$ (Fig. 2c). The heterogeneity test and $\mathrm{I}^{2}$ ( $p$-value $=0.174$ and $\mathrm{I}^{2}=39.6 \%$ ) showed no significant between-studyvariation. However, definitive conclusions could not be drawn from these treatment differences due to the small number of studies $(N=4)$ evaluated, which also explains the wider CIs for the results of the random effects model. In children, the small number of studies with disparate endpoints and results did not allow for meta-analysis.

Sensitivity analyses were performed for $\mathrm{FEV}_{1}$, the high/high-medium dose subgroup data for morning PEF, and $\mathrm{FEF}_{25-75 \%}$, by excluding trials with crossover design [23] or with multiple arms (only in the case of $\mathrm{FEV}_{1}$ ) [25]. The results of the sensitivity analyses for $\mathrm{FEV}_{1}$ and 
a) $\operatorname{FEV}_{1}(\mathrm{~L})$ change from baseline between treatments

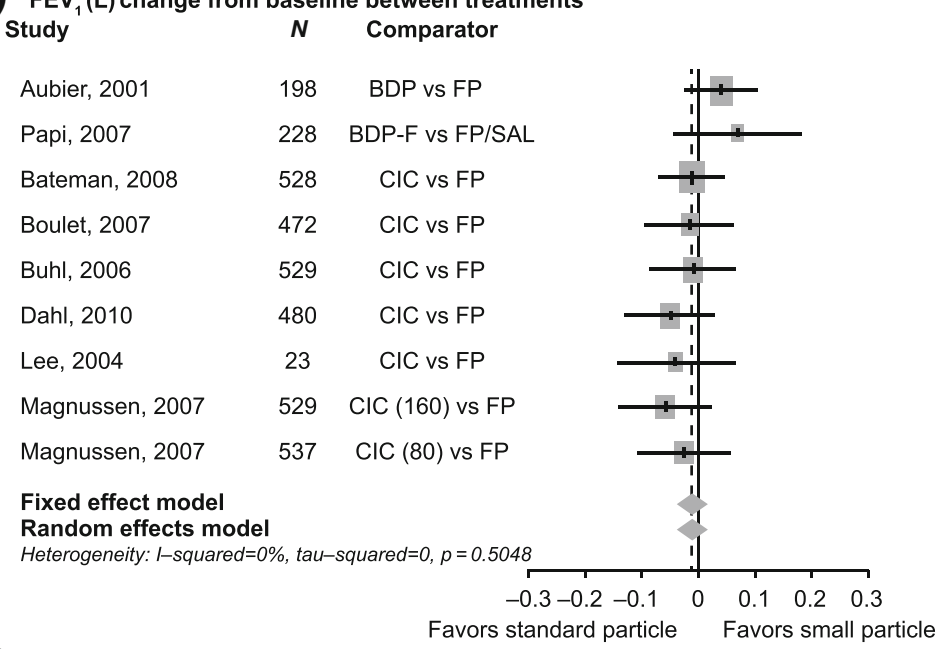

Mean difference $(95 \% \mathrm{Cl})$

$0.040 \quad[-0.022,0.102]$

$0.070 \quad[-0.043,0.183]$

$-0.013 \quad[-0.070,0.044]$

$-0.015 \quad[-0.093,0.063]$

$-0.010 \quad[-0.086,0.066]$

$-0.050 \quad[-0.128,0.028]$

$-0.040 \quad[-0.144,0.064]$

$-0.059 \quad[-0.140,0.022]$

$-0.025[-0.106,0.056]$

$-0.011 \quad[-0.037,0.014]$

$-0.011 \quad[-0.037,0.014]$

Favors standard particle Favors small particle

b) Morning PEF (L/min) change from baseline between treatments

i) Medium/low dose levels

Study

N Comparator

Fairfax, 2001

172 BDP vs FP

Thongngarm, 2005

Papi, 2007

Boulet, 2007

30 BDP vs FP

Buhl, 2006

228 BDP-F vs FP/SAL

472 CIC vs FP

$529 \quad \mathrm{CIC}$ vs FP

Dahl, 2010

$480 \quad \mathrm{ClC}$ vs FP

Fixed effect model

Random effects model

Heterogeneity: $I$-squared $=42.3 \%$, tau-squared $=30.58, p=0.1231$

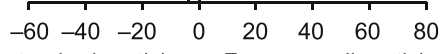

Favors standard particle Favors small particle

ii) High/high-medium dose levels
Study

Aubier, 2001

Bateman, 2008

Lee, 2004
Comparator

$\begin{array}{cc}198 & \text { BDP vs FP } \\ 528 & \text { CIC vs FP } \\ 23 & \text { CIC vs FP }\end{array}$

Fixed effect model

Random effects mode

Heterogeneity: $I$-squared $=0 \%$, tau-squared $=0, p=0.5035$

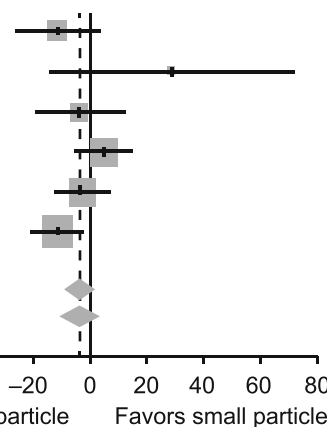

Mean difference $(95 \% \mathrm{Cl})$

$-11.500 \quad[-26.480,3.480]$

$29.050 \quad[-14.291,72.391]$

$-3.850 \quad[-19.726,12.026]$

$4.900 \quad[-5.357,15.157]$

$-3.000 \quad[-12.977,6.977]$

$-11.500 \quad[-20.712,-2.288]$

$-4.223 \quad[-9.205,0.759]$

$-3.874[-10.915,3.166]$

Mean difference $(95 \% \mathrm{CI})$

$12.460 \quad[-2.282,27.202]$

$5.300 \quad[-7.048,17.648]$

$1.000 \quad[-11.286,13.286]$

$5.551 \quad[-1.948,13.049]$

$5.551 \quad[-1.948,13.049]$

\section{0}

Favors standard particle Favors small particle

C) $\operatorname{FEF}_{25 \%-75 \%}(\%$ predicted $)$ change from baseline between treatments

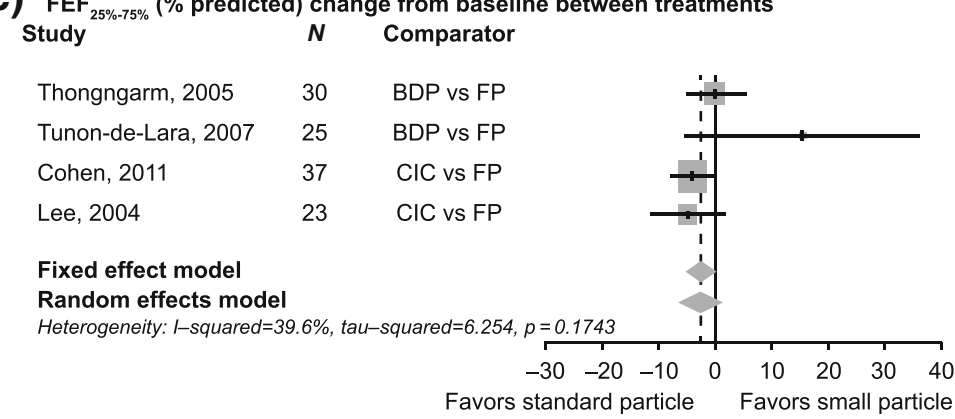

Mean difference $(95 \% \mathrm{Cl})$

$-0.100 \quad[-5.263,5.063]$

$15.300 \quad[-5.352,35.952]$

$-4.200 \quad[-7.931,-0.469]$

$-5.000 \quad[-11.616,1.616]$

$-2.853 \quad[-5.579,-0.127]$

$-2.418 \quad[-6.400,1.564]$

$\begin{array}{cc}\begin{array}{c}\text { Weight, \% } \\ \text { (fixed) }\end{array} & \begin{array}{c}\text { Weight, \% } \\ \text { (random) }\end{array} \\ 16.7 & 16.7 \\ 5.1 & 5.1 \\ 20.0 & 20.0 \\ 10.5 & 10.5 \\ 11.3 & 11.3 \\ 10.5 & 10.5 \\ 6.0 & 6.0 \\ 9.9 & 9.9 \\ 9.9 & 9.9 \\ \mathbf{1 0 0} & -- \\ -- & \mathbf{1 0 0}\end{array}$

Weight, \% (fixed)

11.1

Weight, \%

11.1

$1.3 \quad 2.5$

$9.8 \quad 13.4$

$23.6 \quad 22.3$

$24.9 \quad 22.8$

$29.2 \quad 24.5$

100

$\overline{--}$

100

Weight, $\% \quad$ Weight, $\%$

(fixed) (random)

$25.9 \quad 25.9$

$36.9 \quad 36.9$

$37.2 \quad 37.2$

100

100

Weight, \% Weight, \%

(fixed) (random)

$27.9 \quad 31.3$

$1.7 \quad 3.5$

$53.4 \quad 41.8$

$17.0 \quad 23.4$

100

Fig. 2 (See legend on next page.) 
(See figure on previous page.)

Fig. 2 Pooled effects for efficacy endpoints with $95 \% \mathrm{Cl}$ of eligible studies comparing small versus standard size particle ICS medications. Mean difference in change from baseline between treatments for (a) FEV 1 , (b) morning PEF and (c) $\mathrm{FEF}_{25 \%-75 \%}$. BDP, beclometasone dipropionate, BDP-F, beclometasone dipropionate/formoterol fumarate; $\mathrm{Cl}$, confidence interval; $\mathrm{ClC}$, ciclesonide; $\mathrm{FEF}_{25-75 \%} \%$ predicted forced expiratory flow between $25 \%$ and $75 \%$ of forced vital capacity; FEV ${ }_{1}$, forced expiratory volume in $1 \mathrm{~s}$; FP, fluticasone propionate; FP/SAL, fluticasone propionate/salmeterol; PEF, peak expiratory flow

the morning PEF were similar to the results of the final model. However, the results of the sensitivity analysis for $\mathrm{FEF}_{25-75 \%}$ (excluding Lee et al. [22]), were found to differ from the final model. There was a statistically significant treatment difference between standard size and small size particles on $\mathrm{FEF}_{25-75 \%}$ in the final fixed effect model $(-2.853 \mathrm{~L} / \mathrm{min} ; 95 \%$ CI $-5.579,-0.127 ; p=0.040)$ but not in the sensitivity analysis $(-2.414 \mathrm{~L} / \mathrm{min} ; 95 \% \mathrm{CI}$ $-5.406,0.578 ; p=0.114)$. These conflicting conclusions may be due to small patient sample sizes.

\section{Benefit-risk plots}

The benefit-risk plots included effect estimates from all individual studies included in the analysis of each efficacy and safety endpoint. For adult/adolescent patients (aged $\geq 12$ years), the effect on total asthma symptom score and rescue medication use was only assessed for FP versus CIC. No clinically meaningful differences were noted across the five efficacy endpoints considered $\left(\mathrm{FEV}_{1}\right.$, morning PEF, and $\mathrm{FEF}_{25-75 \%}$, asthma symptoms and rescue medication use) in both adults (Fig. 3) and children (Fig. 4).

No appreciable differences were noted for most safety endpoints (AEs, local steroid effects, upper respiratory tract infections, growth and bone metabolism, and adrenal suppression) in both adults and children (Figs. 3 and 4); most studies though were not designed to test treatment differences for safety endpoints. Patients receiving FP experienced more local steroid effects at the upper airways than those receiving $\mathrm{CIC}$; this was likely due to the fact that CIC is administered as a pro-drug which is only activated in the lower airways. The cortisol levels data were variable, with no clear differentiation between treatments.

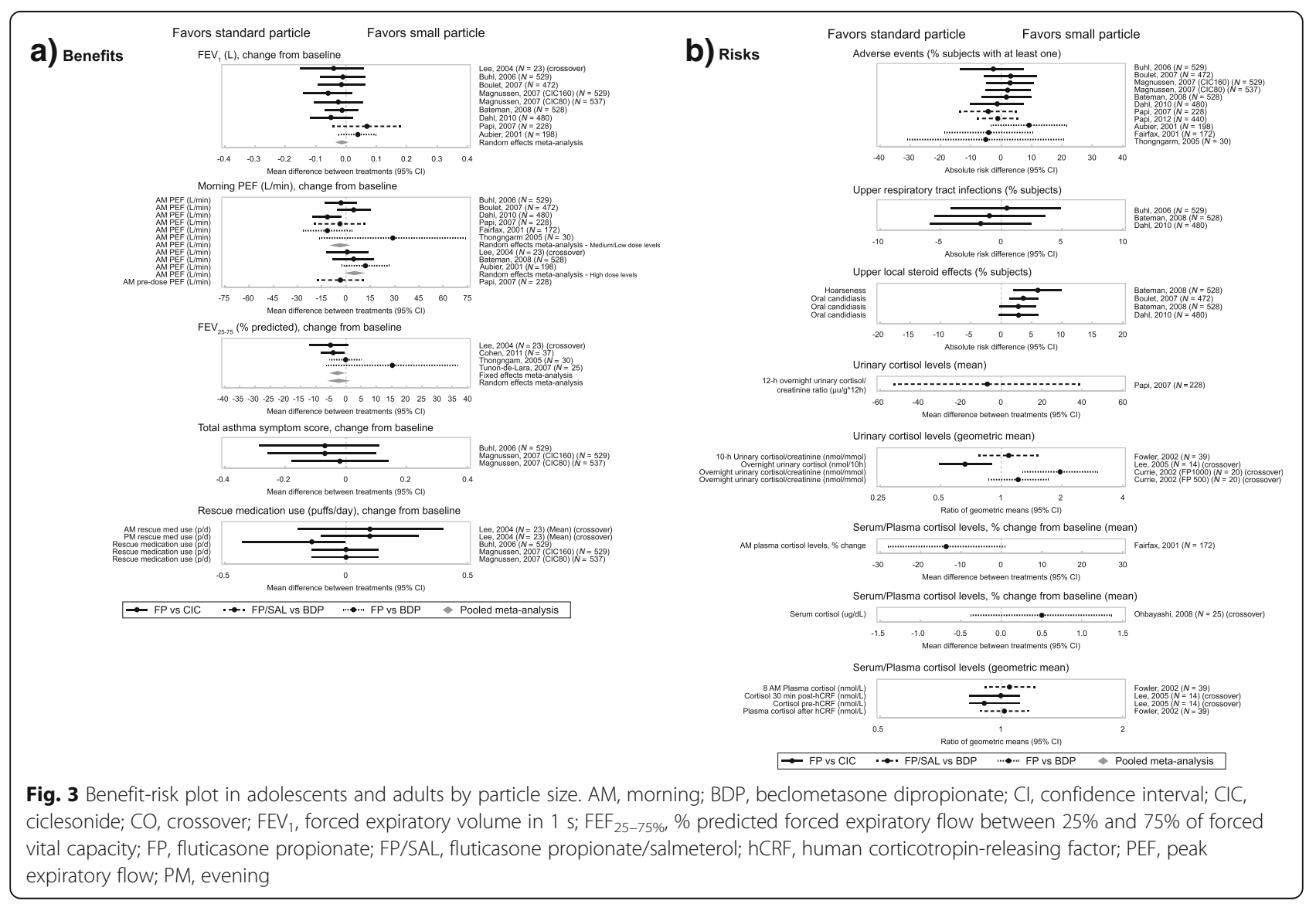




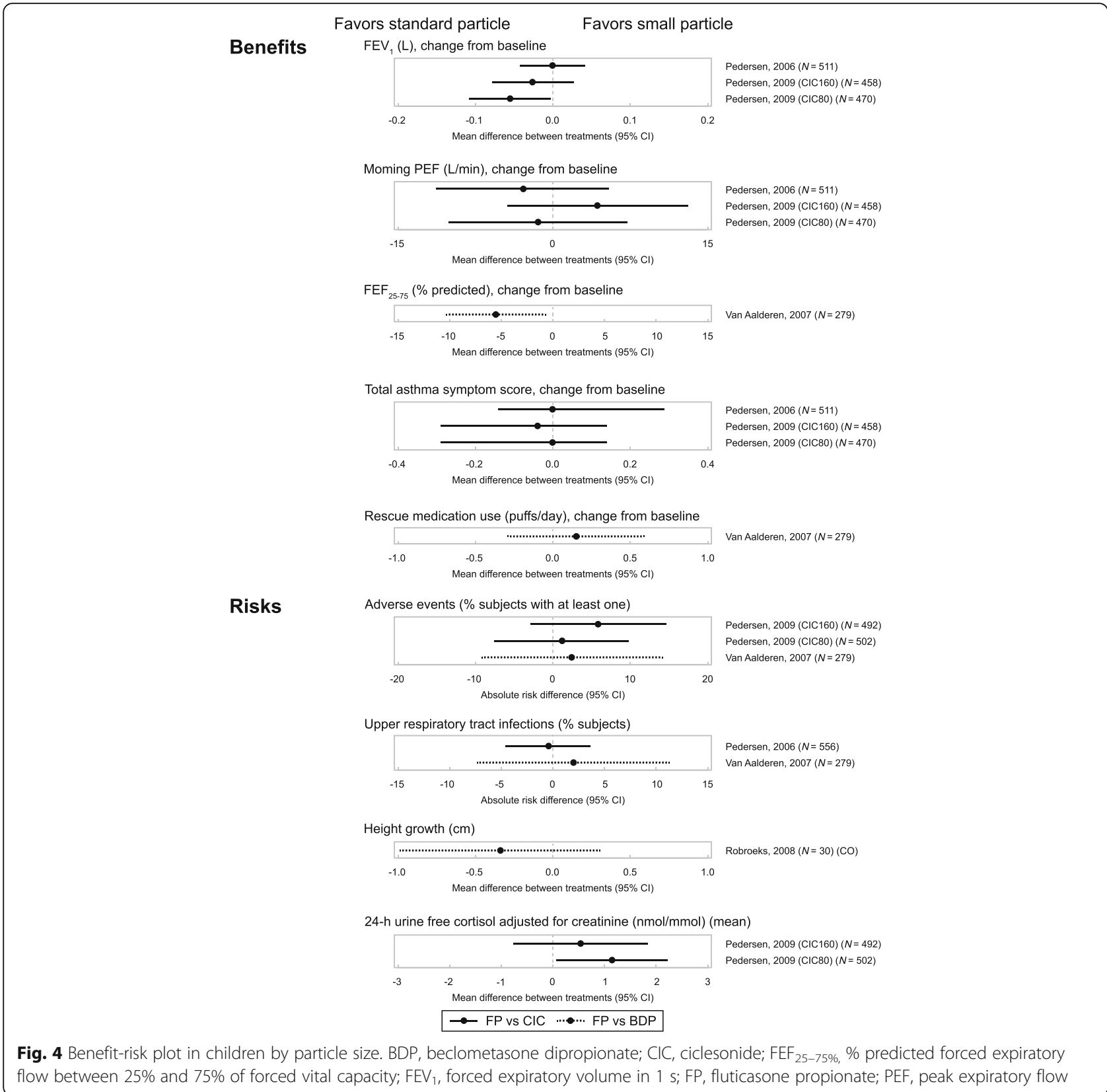

Publication bias Based on the funnel plots and the test of asymmetry ( $p$-values ranging from 0.225-0.822) (Fig. 5), the $\mathrm{FEV}_{1}$, morning $\mathrm{PEF}$ and $\mathrm{PEF}_{25-75 \%}$ data did not exhibit asymmetry, which suggests that there is neither publication bias nor a systematic difference between smaller and larger studies ('small study effects').

\section{Discussion}

\section{Summary}

In this meta-analysis of studies in adults and adolescents, no significant differences were observed between standard size (FP and FP/SAL) and small size (BDP, CIC and BDP-F) particles ICS for change in
$\mathrm{FEV}_{1}$, morning PEF or $\mathrm{FEF}_{25-75 \%}$ using the random effects model. Similarly, no significant differences were observed between standard size and small size particles ICS in the subgroup analysis according to dose for morning PEF. However, it was observed that the result of the medium/low dose analysis for morning PEF was slightly in favor of standard particles (i.e. standard size particles ICS demonstrated better efficacy) versus small particles while the converse was true for high/high-medium dose analysis. This observed difference in the subgroup analyses suggests that dose level may be the effect modifier for morning PEF. 


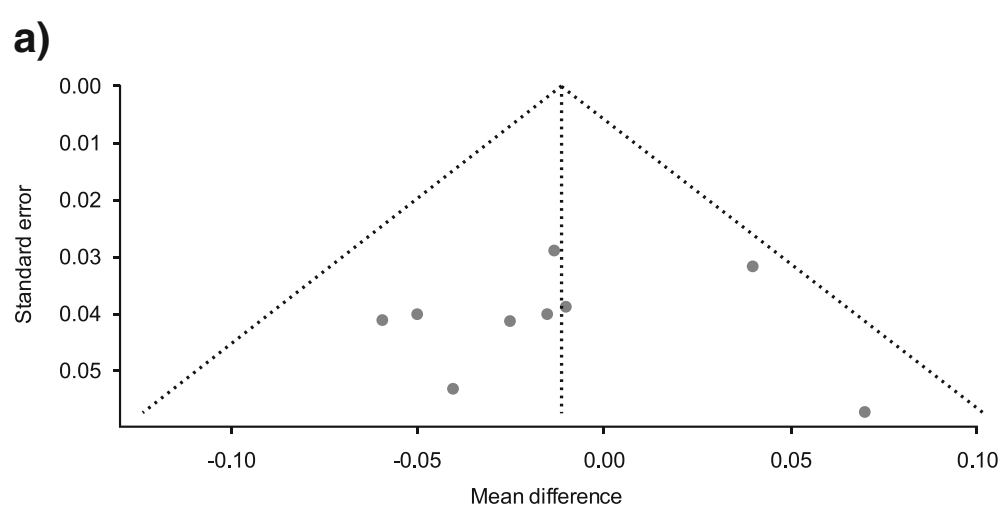

b)

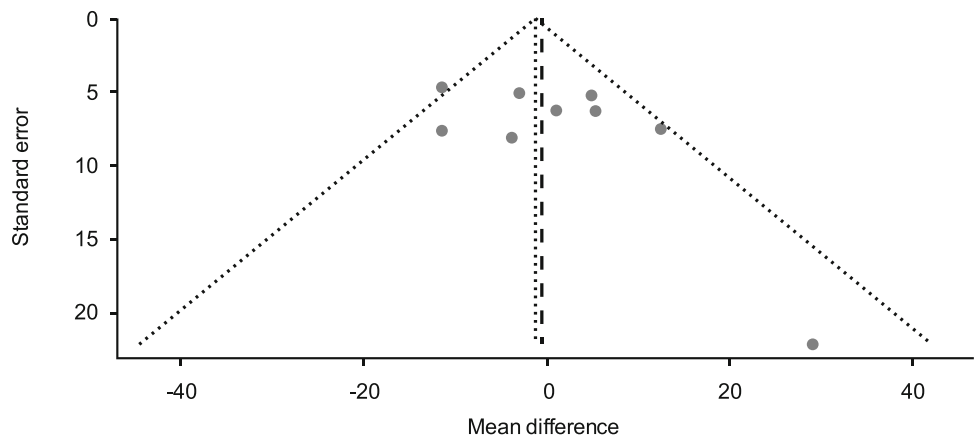

c)

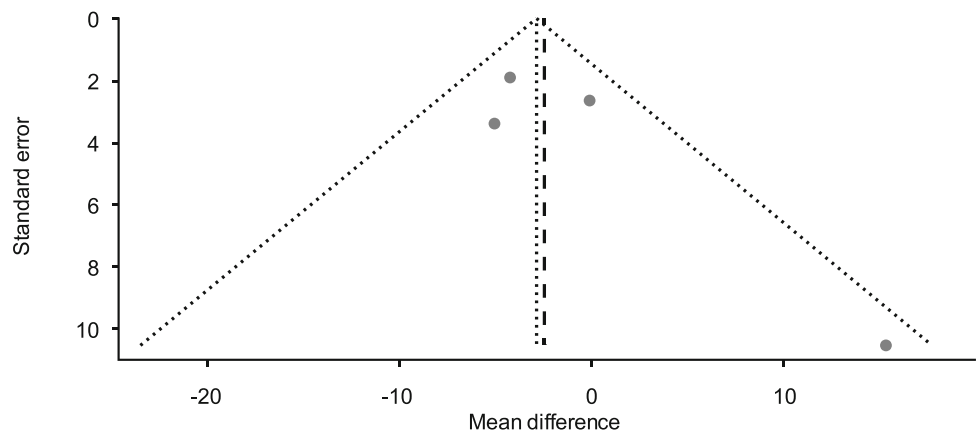

Fig. 5 Funnel plots for studies included in the (a) $\mathrm{FEV}_{1}$, (b) morning PEF and (c) $\mathrm{FEF}_{25-75 \%}$ endpoints meta-analyses. a. Linear regression test of funnel plot asymmetry: $t=-0.2342, d f=7, p$-value $=0.822$. b. Linear regression test of funnel plot asymmetry: $t=1.332, d f=7, p$-value $=0.225$. c. Linear regression test of funnel plot asymmetry $\mathrm{t}=1.547, \mathrm{df}=6, \mathrm{p}$-value $=0.262$. $\mathrm{FEF}_{25-75 \%} \%$ predicted forced expiratory flow between $25 \%$ and $75 \%$ of forced vital capacity; FEV 1 , forced expiratory volume in 1 s; PEF, peak expiratory flow

$\mathrm{FEF}_{25-75 \%}$ was chosen as an efficacy endpoint as it is a more sensitive indicator for disease in the small airways than $\mathrm{FEV}_{1}$ [9], and thus more likely to demonstrate variations in efficacy if the smaller particles were meeting the small airways. However, there is ongoing debate as to the role of $\mathrm{FEV}_{25-75 \%}$ values in assessing asthma control and phenotype-driven treatment [33, 34]. Although there were no significant differences in $\mathrm{FEF}_{25-75 \%}$ for different particle sizes using the random effect model (mostly due to the small number of studies), a statistically significant difference in favor of standard particles was seen when the fixed effects model was used because the heterogeneity tests were not significant. However, this statistical difference was not clinically significant.

In terms of specific treatment comparisons, little or no differences in $\left(\mathrm{FEV}_{1}\right.$ and $\left.\mathrm{PEF}\right)$ were reported for FP versus CIC, FP/SAL versus BDP-F, and FP versus BDP. There were no significant differences in $\mathrm{FEF}_{25-75 \%}$ for $\mathrm{FP}$ versus $\mathrm{CIC}$; however, evidence of increased efficacy in $\mathrm{FEF}_{25-75 \%}$ was demonstrated in one trial with FP versus BDP [18] while the opposite was shown in another [16]. The results of this study also showed no significant 
differences in asthma symptoms and use of rescue medications between small and standard size ICS. However, these results should be treated with caution as these parameters were only evaluated between FP and CIC and not for FP versus BDP.

For the safety endpoints considered, no appreciable differences were observed for most endpoints though it should be noted that the majority of studies were not designed to test treatment differences for safety endpoints. Adult/adolescent patients experienced more local steroid effects with standard particles than small particles. The observation that the local steroid effects at the upper airways favored small particles was likely due to the fact that CIC is administered as a pro-drug which is only activated in the lower airways. Overall, the cortisol data were variable with no clear differentiations between particle sizes.

\section{Particle size, lung deposition, and clinical outcomes}

Although there is a relationship between smaller particle size and increased delivery to the distal lung $[35,36]$, the current study demonstrated that increased deposition in the distal lung does not appear to translate into improved clinical outcomes for patients with asthma. These results are unsurprising for a number of reasons, not least because an increased proportion of the inhaled dose is likely to deposit in the distal respiratory compartment beyond the conducting airways. Based on current understanding, the effect of using an aerosol with smaller particle size or a 'finer' aerosol is both to increase the dose reaching the lungs by reducing the oropharyngeal deposition and to increase the proportion of that dose depositing distally. While simplistically this increased distal deposition might be considered to result in increased deposition in the 'small airways', it is important to recognize the increased deposition in fact occurs in the respiratory compartment beyond the conducting airways.

In considering the impact of ICS with different particle sizes it is important to understand the factors influencing the pattern of deposition of an aerosol in the complex 3D structure formed by the conducting airways (large and small) and pulmonary/alveolar compartments of the lung. Current mathematical modeling methods combined with 3D imaging suggest that the vast majority of inhaled aerosol $(>90 \%)$ inhaled during tidal breathing is delivered to the pulmonary/alveolar compartment beyond the conducting airways, and this will increase with the modified inspiratory breath when using a pMDI (with or without a holding chamber) $[37,38]$. Hence for a given dose administered to the conducting airways (large, medium, and small), the dose delivered more distally will be relatively greater with the 'finer' aerosol. With such a small proportion of the aerosol depositing in the conducting airways it is unlikely that there will be a major change in the concentration of aerosol at the epithelial surface of the conducting airways. This is particularly true of the small conducting airways which have a much greater relative surface area than the more central airways.

Another point of consideration is the conjecture that 'finer' aerosols will penetrate more effectively in the face of airways narrowing. However, any perceived advantage of finer aerosols in accessing the blocked/narrowed airways would be transient in nature as it has been shown that the blockage is resolved very rapidly in the vast majority of patients with asthma when they commence ICS treatment [39].

Based on the results of this study and discussions above, it is evident that the key issue should be the evaluation of the 'therapeutic index' of different drugdevice combinations rather than a comparison of aerosol particle sizes for controlling asthma. Unfortunately, there is no robust method for assessing this. As previously mentioned, apart from particle size, drug deposition within the lung is dependent on other factors such as inhaler device and inhalation technique, which varies between patients [36, 40, 41]. Thus, these factors make it impossible to know what lung doses will be achieved when an individual patient uses a particular drug-device combination, even under controlled conditions [42]. Consequently current guidelines advocate titration of ICS dosages against symptoms and spirometric data [2]. Using the lowest effective dose ensures maximum efficacy and minimizes the risk of side effects. The guidelines do not distinguish between corticosteroids formulations and this approach is supported by this systematic review.

A number of observational studies and historical matched cohort analyses have recently been published comparing the outcomes and cost of treatment with small versus standard size particle ICS in patients with asthma [43-46]. In general, the studies found that asthma treatment outcomes were similar or better with small size particle ICS (BDP) compared with standard size particle ICS (FP). However, such studies are usually confounded by variables that are not present in RCTs. For example, observational studies often rely on prescription data (which does not necessarily translate to actual dosage taken) or are not able to quantify past exposure of some drugs. Furthermore, control for asthma severity in these studies was often indirect via rescue medication and hospitalizations. Another factor that might confound the results of these studies is the lack of patient randomization.

Most of the individual clinical studies included in this review may not have been powered to detect clinically meaningful differences but statistically significant differences. This meta-analysis offered the opportunity to increase the sample size and power to calculate pooled estimates for treatment differences. Despite this increased power to detect statistical differences, the relevance and 
clinical meaningfulness of these results must be determined beyond the results showing statistical significance.

A potential limitation of literature reviews/meta-analyses pertains to publication bias. Searches of databases such as PubMed or EMBASE yield long lists of studies that have been published. Such searches are unlikely to yield a representative sample because studies that show a 'positive' result are more likely to be published than those that do not. However, based on the funnel plots and the test of asymmetry, the $\mathrm{FEV}_{1}$, morning PEF and $\mathrm{FEF}_{25-75 \%}$ data did not exhibit asymmetry, which suggests that publication bias is not likely to be a limiting factor in this study.

Another potential limitation is that the present study did not explore whether other parameters of inflammation such as fractional exhaled nitric oxide [47] were differentially affected by particle size. Similarly, endpoints such as asthma exacerbations, which together with lung function and asthma symptoms indicate sub-optimal asthma control [1], was also not assessed in the present study.

\section{Conclusion}

In summary, the results of this systematic review do not support the suggestion that smaller size particle ICS are intrinsically more 'effective' than larger standard size particle ICS on the endpoints of lung function, asthma symptoms and rescue medication use. Markers of inflammation and asthma exacerbation were not assessed in this meta-analysis and so the ability of small particle treatments to differentially affect these outcomes were not possible to ascertain. No study to date has clearly addressed the key issue of the relative therapeutic index of the different drug-delivery combinations though there are robust data that regular ( $>80 \%$ of doses) use of these treatments at licenced doses is effective and well tolerated.

\section{Abbreviations}

AE: Adverse event; AM: Morning; BDP: Beclometasone dipropionate; BDP-F: Beclometasone dipropionate-formoterol; CFC: Chlorofluorocarbon; $\mathrm{Cl}$ : Confidence interval; CIC: Ciclesonide; CL: Clearance; CO: crossover; CV-VC: Closing volume/vital capacity; DPI: Dry powder inhaler; $\mathrm{dx}$ : Diagnosis; ER: Emergency room; FEF: Forced expiratory flow; $\mathrm{FEV}_{1}$ : Forced expiratory volume in $1 \mathrm{~s}$; FP: Fluticasone propionate; FP/ SAL: Fluticasone propionate-salmeterol combination; FVC: Forced vital capacity; hCRF: human corticotropin-releasing factor;

HFA: Hydrofluoroalkane; HFA-pMDI: Hydrofluoroalkane-pressurized metered dose inhaler; ICS: Inhaled corticosteroids; ITT: Intent-to-treat; MMAD: Mass median aerodynamic diameter; NO: Nitric oxide; NR: Not reached; PEF: Peak expiratory flow; PEFR: Peak expiratory flow rate; PM: Evening; PMDI: Pressurized metered dose inhalers; PP: Per protocol; ppb: Part per billion; RCT: Randomized controlled trial; RV: Residual volume; SABA: Short-acting beta agonists; SE: Standard error; SVC: Slow vital capacity; TGV: Thoracic gas volume; TLC: Total lung capacity; Tx: Treatment

\section{Acknowledgments}

All authors meet the criteria for authorship set forth by the International Committee for Medical Journal Editors. The authors wish to acknowledge the following individuals for their critical review during the development of the outlines and first draft of this manuscript: David Hinds, Daniel Parks, and
Michael Gibbs (all employees of GSK). The authors also acknowledge Maggie Davis (GSK employee) for editorial input, data review, and coordination of author review cycles. Initial drafting of the outline was conducted by Tracy Taylor Sandell of TaylorWrit Communications Ltd, UK, funded by GSK. Editorial support in the form of editorial suggestions to draft versions of this paper, assembling tables and figures, collating author comments, copyediting, and referencing, was provided by Karen Yee PhD, of Fishawack Indicia Ltd, UK, and was funded by GSK.

\section{Funding}

Financial support for the conduct of this study and preparation of the article was provided by GSK. Staff from GSK were involved in the study design, analysis and interpretation of data, in the preparation of the article, and in the decision to submit the article for publication.

\section{Availability of data and materials}

The dataset supporting the conclusions of this article is available on the GSK Study Register http://www.gsk-clinicalstudyregister.com/study/202012\#rs.

\section{Authors' contributions}

RDS participated in study conception and design, data acquisition and interpretation. CEB participated in study conception and design, data acquisition (involved in meta-analyses), analysis and interpretation. RA-C contributed to the study concept and design, and data acquisition (conducted meta-analyses). EAS participated in data acquisition (planned and conducted literature review, wrote literature review report, performed preliminary data extraction, critically reviewed material). DS participated in study conception and design; data acquisition, analysis and interpretation. MLE contributed to data analysis and interpretation. NB participated in study conception and design; data acquisition, analysis and interpretation. All authors read and approved the final manuscript.

\section{Competing interests}

CEB was contracted by GSK during the conduct of this analysis. RDS was a full-time employee of GSK during the conduct of this analysis and is currently a shareholder. RA-C, DS, and NB are full-time employees and shareholders of GSK. MLE previously received honoraria for presentations/attending advisory boards as well as support for attending conferences from a number of companies producing inhaled corticosteroid products including GSK, AstraZeneca, $3 \mathrm{M}$, Orion, and Chiesi. EAS does not have any conflict of interest to declare.

\section{Consent for publication}

Not applicable.

Ethics approval and consent to participate Not applicable.

\section{Author details}

${ }^{1}$ GSK, Middlesex, Stockley Park, Uxbridge, UK. ${ }^{2}$ GSK, Research Triangle Park, NC, USA. ${ }^{3}$ GSK, Upper Providence, PA, USA. ${ }^{4}$ University of Washington, School of Medicine, Seattle, WA, USA. ${ }^{5}$ New England Research Institutes, Watertown, MA, USA. ${ }^{6}$ The University of Western Australia, Crawley, WA 6009, Australia. ${ }^{7}$ GSK, Brentford, UK. ${ }^{8}$ William Harvey Research Institute, Barts and The London School of Medicine and Dentistry, London, UK. ${ }^{9}$ Current affiliation: Janssen Pharmaceuticals, Titusville, NJ, USA. ${ }^{10} \mathrm{Current}$ affiliation: University of North Carolina at Chapel Hill, Chapel Hill, NC, USA. ${ }^{11}$ PHASTAR, Chiswick, London, UK.

Received: 11 June 2016 Accepted: 12 December 2016

Published online: 07 February 2017

\section{References}

1. Global Strategy for Asthma Management and Prevention. Available from: http://ginasthma.org/gina-reports/. Accessed May 2016.

2. British guideline on the management of asthma. Available from: https:// www.brit-thoracic.org.uk/standards-of-care/guidelines/btssign-britishguideline-on-the-management-of-asthma/. Accessed May 2016.

3. Usmani OS. Small-airway disease in asthma: pharmacological considerations. Curr Opin Pulm Med. 2015;21(1):55-67. 
4. Cohen J, Postma DS, Douma WR, et al. Particle size matters: diagnostics and treatment of small airways involvement in asthma. Eur Respir $\mathrm{J}$. 2011;37(3):532-40

5. Dyer MJ, Halpin DM, Stein K. Inhaled ciclesonide versus inhaled budesonide or inhaled beclomethasone or inhaled fluticasone for chronic asthma in adults: a systematic review. BMC Fam Pract. 2006;7:34.

6. Grainger $\mathrm{Cl}$, Saunders M, Buttini F, et al. Critical characteristics for corticosteroid solution metered dose inhaler bioequivalence. Mol Pharm. 2012;9(3):563-9.

7. Buttini F, Miozzi M, Balducci AG, et al. Differences in physical chemistry and dissolution rate of solid particle aerosols from solution pressurised inhalers. Int J Pharm. 2014;465(1-2):42-51.

8. Gentile DA, Skoner DP. New asthma drugs: small molecule inhaled corticosteroids. Curr Opin Pharmacol. 2010;10(3):260-5.

9. Lipworth B. Targeting the small airways asthma phenotype: if we can reach it, should we treat it? Ann Allergy Asthma Immunol. 2013;110(4):233-9.

10. Cochrane Handbook for Systematic Reviews of Interventions Version 5.1.0 [updated March 2011]. The Cochrane Collaboration, 2011. Available from: http://community.cochrane.org/handbook. Accessed May 2016.

11. Aubier M, Wettenger R, Gans SJ. Efficacy of HFA-beclomethasone dipropionate extra-fine aerosol (800 microg day(-1)) versus HFA-fluticasone propionate (1000 microg day(-1)) in patients with asthma. Respir Med. 2001;95(3):212-20.

12. Currie GP, Fowler SJ, Wilson AM, et al. Airway and systemic effects of hydrofluoroalkane fluticasone and beclomethasone in patients with asthma. Thorax. 2002;57(10):865-8.

13. Fairfax A, Hall I, Spelman R. A randomized, double-blind comparison of beclomethasone dipropionate extrafine aerosol and fluticasone propionate. Ann Allergy Asthma Immunol. 2001;86(5):575-82.

14. Ohbayashi $H$, Adachi M. Hydrofluoroalkane-beclomethasone dipropionate effectively improves airway eosinophilic inflammation including the distal airways of patients with mild to moderate persistent asthma as compared with fluticasone propionate in a randomized open double-cross study. Allergol Int. 2008;57(3):231-9.

15. Robroeks $C M$, van de Kant KD, van Vliet $D$, et al. Comparison of the antiinflammatory effects of extra-fine hydrofluoroalkane-beclomethasone vs fluticasone dry powder inhaler on exhaled inflammatory markers in childhood asthma. Ann Allergy Asthma Immunol. 2008;100(6):601-7.

16. Thongngarm T, Silkoff PE, Kossack WS, et al. Hydrofluoroalkane-134A beclomethasone or chlorofluorocarbon fluticasone: effect on small airways in poorly controlled asthma. J Asthma. 2005;42(4):257-63.

17. Tunon-de-Lara JM, Laurent F, Giraud V, et al. Air trapping in mild and moderate asthma: effect of inhaled corticosteroids. J Allergy Clin Immunol. 2007;119(3):583-90.

18. van Aalderen WM, Price D, De Baets FM, et al. Beclometasone dipropionate extrafine aerosol versus fluticasone propionate in children with asthma. Respir Med. 2007;101(7):1585-93.

19. Bateman ED, Linnhof $A E$, Homik $L$, et al. Comparison of twice-daily inhaled ciclesonide and fluticasone propionate in patients with moderate-to-severe persistent asthma. Pulm Pharmacol Ther. 2008:21(2):264-75.

20. Boulet LP, Bateman ED, Voves $R$, et al. A randomized study comparing ciclesonide and fluticasone propionate in patients with moderate persistent asthma. Respir Med. 2007;101(8):1677-86.

21. Buhl R, Vinkler I, Magyar $P$, et al. Comparable efficacy of ciclesonide once daily versus fluticasone propionate twice daily in asthma. Pulm Pharmacol Ther. 2006;19(6):404-12.

22. Dahl R, Engelstatter R, Trebas-Pietras E, et al. A 24-week comparison of low-dose ciclesonide and fluticasone propionate in mild to moderate asthma. Respir Med. 2010;104(8):1121-30.

23. Lee DK, Haggart K, Currie GP, et al. Effects of hydrofluoroalkane formulations of ciclesonide 400 microg once daily vs fluticasone 250 microg twice daily on methacholine hyper-responsiveness in mild-to-moderate persistent asthma. Br J Clin Pharmacol. 2004;58(1):26-33.

24. Lee DK, Fardon TC, Bates CE, et al. Airway and systemic effects of hydrofluoroalkane formulations of high-dose ciclesonide and fluticasone in moderate persistent asthma. Chest. 2005;127(3):851-60

25. Magnussen $H_{1}$ Hofman J, Staneta $P$, et al. Similar efficacy of ciclesonide once daily versus fluticasone propionate twice daily in patients with persistent asthma. J Asthma. 2007:44(7):555-63.

26. Pedersen S, Garcia Garcia ML, Manjra A, et al. A comparative study of inhaled ciclesonide $160 \mathrm{microg} /$ day and fluticasone propionate 176 microg/ day in children with asthma. Pediatr Pulmonol. 2006;41(10):954-61.
27. Pedersen S, Engelstatter R, Weber HJ, et al. Efficacy and safety of ciclesonide once daily and fluticasone propionate twice daily in children with asthma. Pulm Pharmacol Ther. 2009;22(3):214-20.

28. van der Molen T, Foster JM, Caeser M, et al. Difference between patientreported side effects of ciclesonide versus fluticasone propionate. Respir Med. 2010;104(12):1825-33.

29. Fowler SJ, Currie GP, Lipworth BJ. Step-down therapy with low-dose fluticasone-salmeterol combination or medium-dose hydrofluoroalkane 134a-beclomethasone alone. J Allergy Clin Immunol. 2002;109(6):929-35.

30. Papi A, Paggiaro P, Nicolini G, et al. Beclomethasone/formoterol vs fluticasone/salmeterol inhaled combination in moderate to severe asthma. Allergy. 2007;62(10):1182-8.

31. Papi A, Nicolini G, Crimi N, et al. Step-down from high dose fixed combination therapy in asthma patients: a randomized controlled trial. Respir Res. 2012;13:54.

32. Scichilone N, Battaglia S, Sorino C, et al. Effects of extra-fine inhaled beclomethasone/formoterol on both large and small airways in asthma. Allergy. 2010;65(7):897-902.

33. Ciprandi G, Cirillo I, Pasotti F, Luigi F, Ricciardolo M. FEF $25-75$ : A marker for small airways and asthma control. Ann Allerg Asthma Im. 2013;111(3):233.

34. Lipworth B. Author response. Ann Allerg Asthma Im. 2013;111(3):234.

35. van den Berge $\mathrm{M}$, ten Hacken $\mathrm{NH}$, Cohen J, et al. Small airway disease in asthma and COPD: clinical implications. Chest. 2011;139(2):412-23.

36. Usmani OS, Barnes PJ. Assessing and treating small airways disease in asthma and chronic obstructive pulmonary disease. Ann Med. 2012;44(2):146-56.

37. Tossici-Bolt L, Fleming JS, Conway JH, et al. An analytical technique to recover the third dimension in planar imaging of inhaled aerosols-2 estimation of the deposition per airway generation. J Aerosol Med. 2007; 20(2):127-40

38. Majoral C, Fleming J, Conway J, et al. Controlled, parametric, individualized, 2D and 3D imaging measurements of aerosol deposition in the respiratory tract of healthy human volunteers: in vivo data analysis. J Aerosol Med Pulm Drug Deliv. 2014:27(5):349-62.

39. Brown HM, Storey G, George WH. Beclomethasone dipropionate: a new steroid aerosol for the treatment of allergic asthma. Br Med J. 1972;1(5800):585-90.

40. Haughney J, Price D, Barnes NC, et al. Choosing inhaler devices for people with asthma: current knowledge and outstanding research needs. Respir Med. 2010;104(9):1237-45.

41. Scheuch $G$, Kohlhaeufl MJ, Brand $P$, et al. Clinical perspectives on pulmonary systemic and macromolecular delivery. Adv Drug Deliv Rev. 2006;58(9-10):996-1008.

42. Everard ML. CFC transition: the emperor's new clothes. Each class of drug deserves a delivery system that meets its own requirements. Thorax. 2000; 55(10):811-4.

43. Colice G, Martin RJ, Israel E, et al. Asthma outcomes and costs of therapy with extrafine beclomethasone and fluticasone. J Allergy Clin Immunol. 2013;132(1):45-54

44. van Aalderen WMC, Grigg J, Guilbert TW, et al. Small-particle inhaled corticosteroid as first-line or step-up controller therapy in childhood asthma. J Allergy Clin Immunol Pract. 2015;3(5):721-31.

45. Price D, Martin RJ, Barnes N, et al. Prescribing practices and asthma control with hydrofluoroalkane-beclomethasone and fluticasone: a real-world observational study. J Allergy Clin Immunol. 2010;126(3):511-8.

46. Lage MJ, Gross GN, Brewster C, et al. Outcomes and costs of patients with persistent asthma treated with beclomethasone dipropionate hydrofluoroalkane or fluticasone propionate. Adv Ther. 2009;26(8):762-75.

47. Dweik RA, Boggs PB, Erzurum SC, et al. An official ATS clinical practice guideline: interpretation of exhaled nitric oxide levels (FENO) for clinical applications. Am J Respir Crit Care Med. 2011:184(5):602-15. 